ISSN 1112-9867

Available online at

http://www.jfas.info

\title{
GEOMETRIC PROPORTIONS IN ISLAMIC ARCHITECTURE: CASE OF THE SIDI EL BENA MOSQUE IN TLEMCEN
}

\author{
W. Hamma \\ Lecturer in Department of Architecture, Faculty of Technology, Abu Beckr Belkaid \\ University of Tlemcen, Algeria
}

Received: 13 Mars 2017 / Accepted: 09 July 2017 / Published online: 01 September 2017

\begin{abstract}
The mosque of Sidi El Benna is one of the Islamic buildings of Tlemcen built up during the Zianid era. In this study its planes, facades and sections were drawn based on different measurements, because this style is characterized by the use of geometric proportions. In the next step the measurements of heights, widths, lengths and angles of different architectural elements were compared in order to find arithmetic and harmonic proportions. The last ones were found especially in the golden number $\Phi$, equal to 1.618. In addition, the cubit of time was computed and found equal to $0.4375 \mathrm{~m}$, almost equal to the Roman one $-0.444 \mathrm{~m}$. Other principles of geometric composition, such as repetition and symmetry, were also identified.
\end{abstract}

Keywords: gold number; Geometric relations; design; Islam; Zianid buildings.

Author Correspondence, e-mail: hammawalid06@hotmail.com

doi: http://dx.doi.org/10.4314/jfas.v9i3.12

\section{INTRODUCTION}

\subsection{Les proportions géométriques dans l'architecture islamique}

L'architecture islamique est basée sur la proportion géométrique rigoureuse que ce soit dans les éléments constructifs (voutes, coupole...), les sculptures (muqarnas et arabesques), la calligraphie ou dans les fresques (Fig.1, 2 ,3), vu que les architectes et artisans musulmans 
étaient d'excellents mathématiciens $[1,2,3]$.

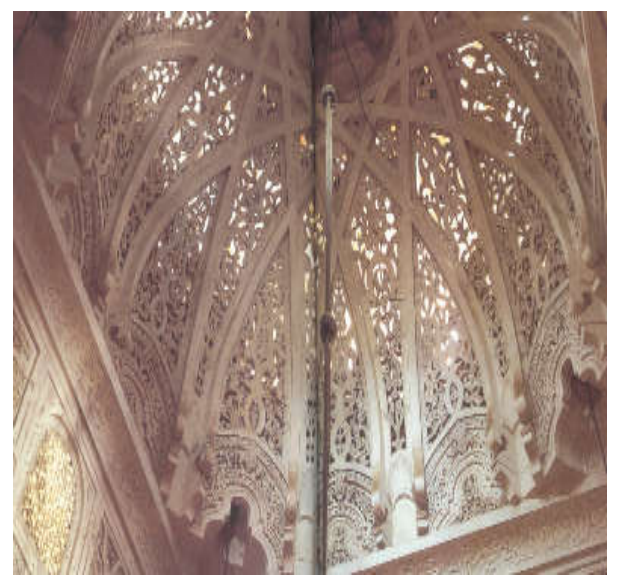

Fig.1. Coupole nervurée de la grande mosquée de Tlemcen

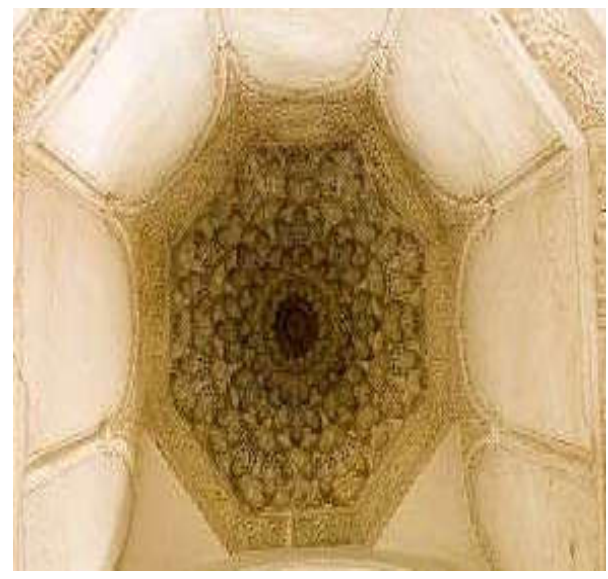

Fig.2. Muqarnas de la mosquée Sidi Bel Hassen de Tlemcen

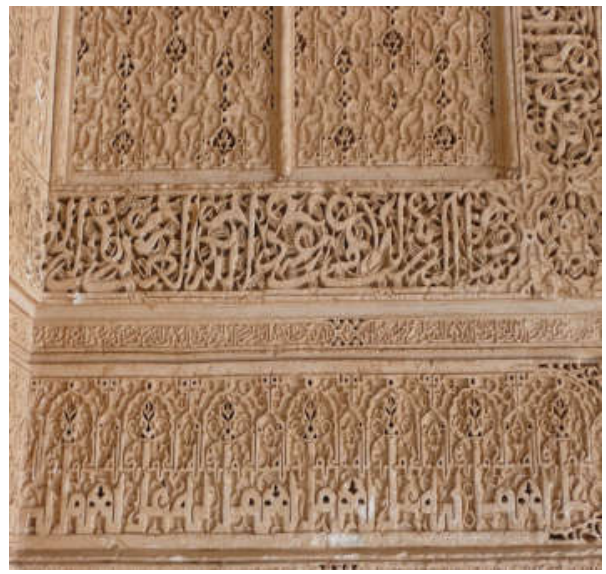

Fig.3. Calligraphie et arabesques de la mosquée Sidi Bel Hassen de Tlemcen

Tout d'abord, elle présente un jeu d'échelle $(1 / 2,1 / 3 \ldots)$, c'est-à-dire un coefficient de 
réduction ou d'agrandissement de tailles des éléments architecturaux $[4,5,6,7]$. Nous citrons l'exemple de la porte principale de la grande mosquée de Nedroma dans la wilaya de Tlemcen qui est égale à la moitié de l'arcade qui l'encadre (Fig.4).

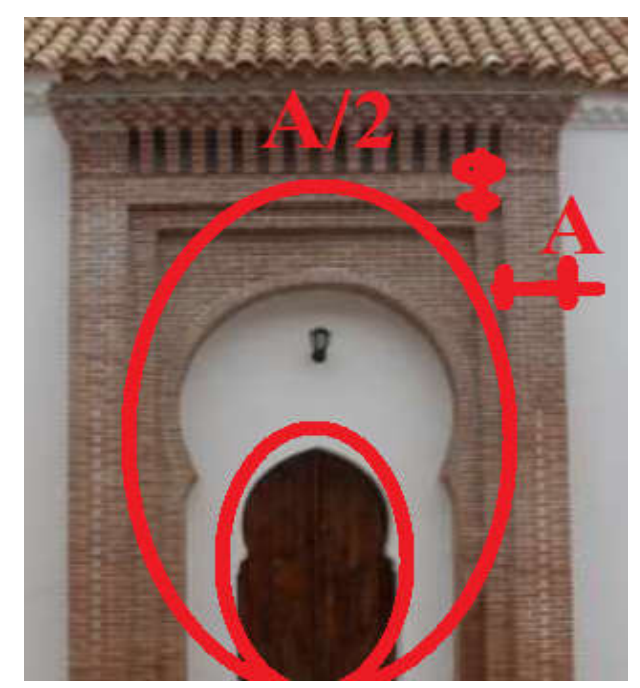

Fig.4. Porte principale de la grande mosquée de Nedroma

Ensuite, la conception par module de base, nous la trouvons au niveau des trames, des arcades, des ouvertures et surtout dans le décor $[8,9,10]$. Ce système numérique repose sur le système de relations proportionnelles. Bien que des différences évidentes soient remarquées dans ce terme, toutes les études conviennent que le module standard, sur lequel repose la majorité des relations proportionnelles, est une dimension dans laquelle les différentes parties du bâtiment sont réitérées (Fig.5). En d'autres termes, c'est un module de dimensions constantes qui lui-même ou ses multiples se réitèrent soit par une augmentation ou une diminution comme base pour toute construction afin d'atteindre finalement la cohérence totale du groupe émergeant, qu'il soit fonctionnel ou esthétique. 


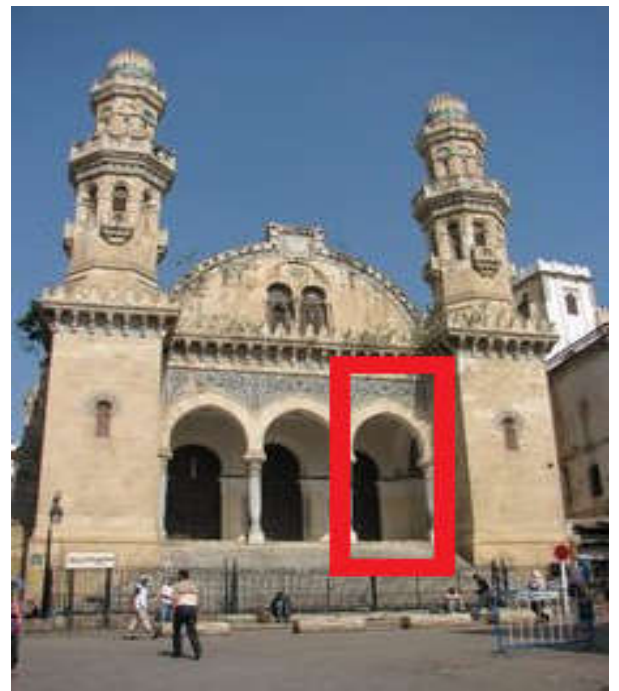

Fig.5. Module de l'arcade du porche d'entrée de la mosquée de Ketchaoua à Alger

En outre, l'utilisation du nombre d'or $(\Phi=1.618)$ dans les différents proportions géométriques $[11,12,13]$. Ce nombre est calculé par plusieurs formules mathématiques. La présence de ce nombre dans les propositions d'une surface rend cette dernière une surface dorée (Fig.6).
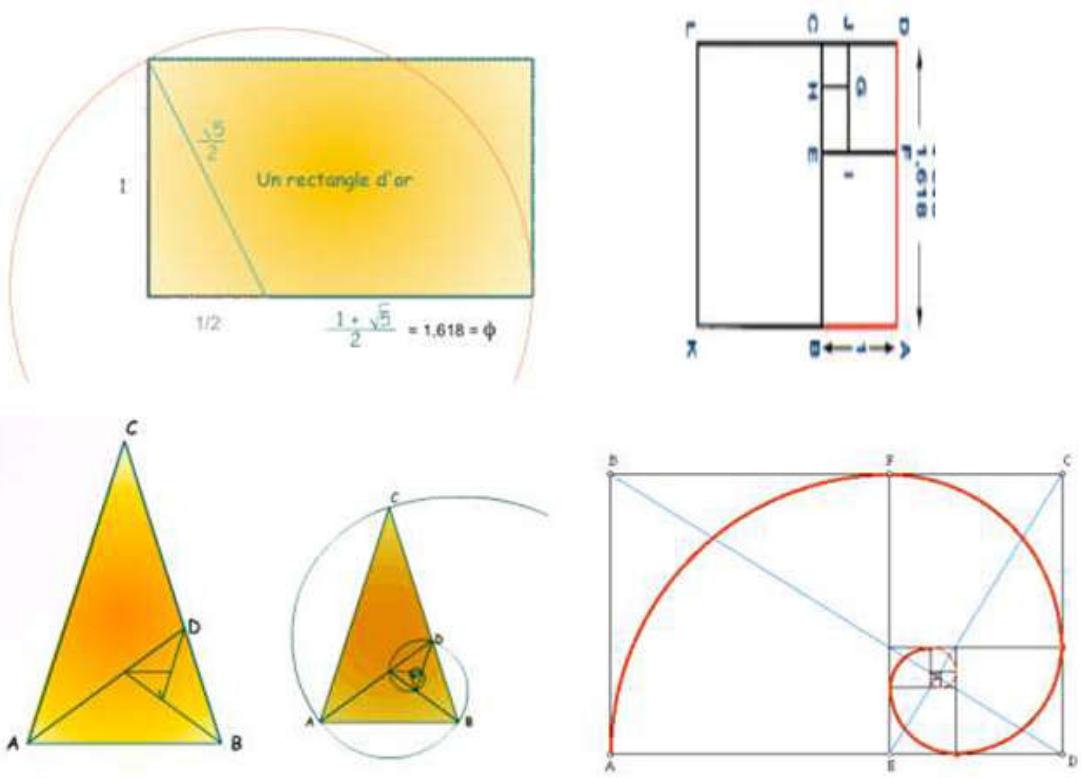

$$
\varphi=2 \cos \left(36^{\circ}\right)
$$

$$
r(\theta)=r \varphi^{2 \theta / \pi}
$$

Fig.6. Surfaces dorées et calcul du nombre d'or [16]

Nous citerons la grande mosquée dont le plan est un rectangle d'or. Le rectangle aussi du patio présente une proportion de $\Phi / 2$ (Fig.7). 


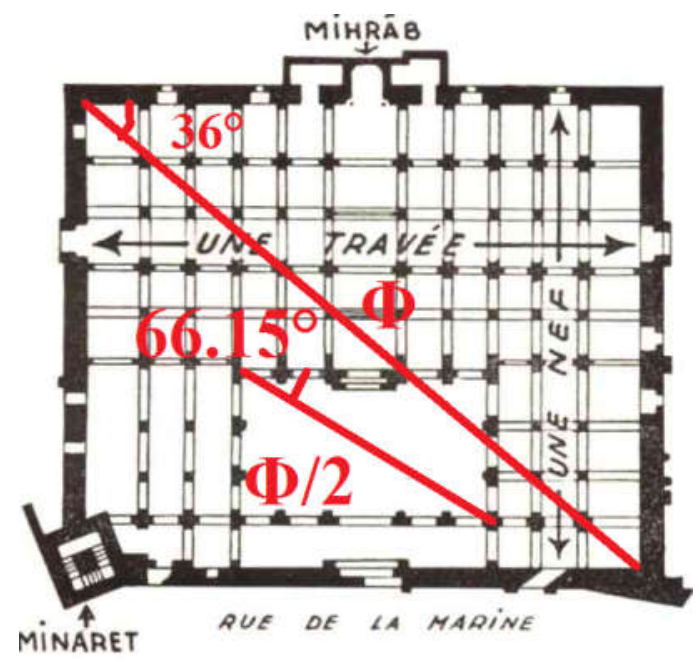

Fig.7. Plan de la grande mosquée d'Alger

Par ailleurs, la rotation des formes de base (Fig.8) pour obtenir d'autres formes plus compliquées tels que le carré pour avoir des étoiles ( 8 branches, $12, \ldots$ ) ou le cercle pour obtenir des entrelacs [14].

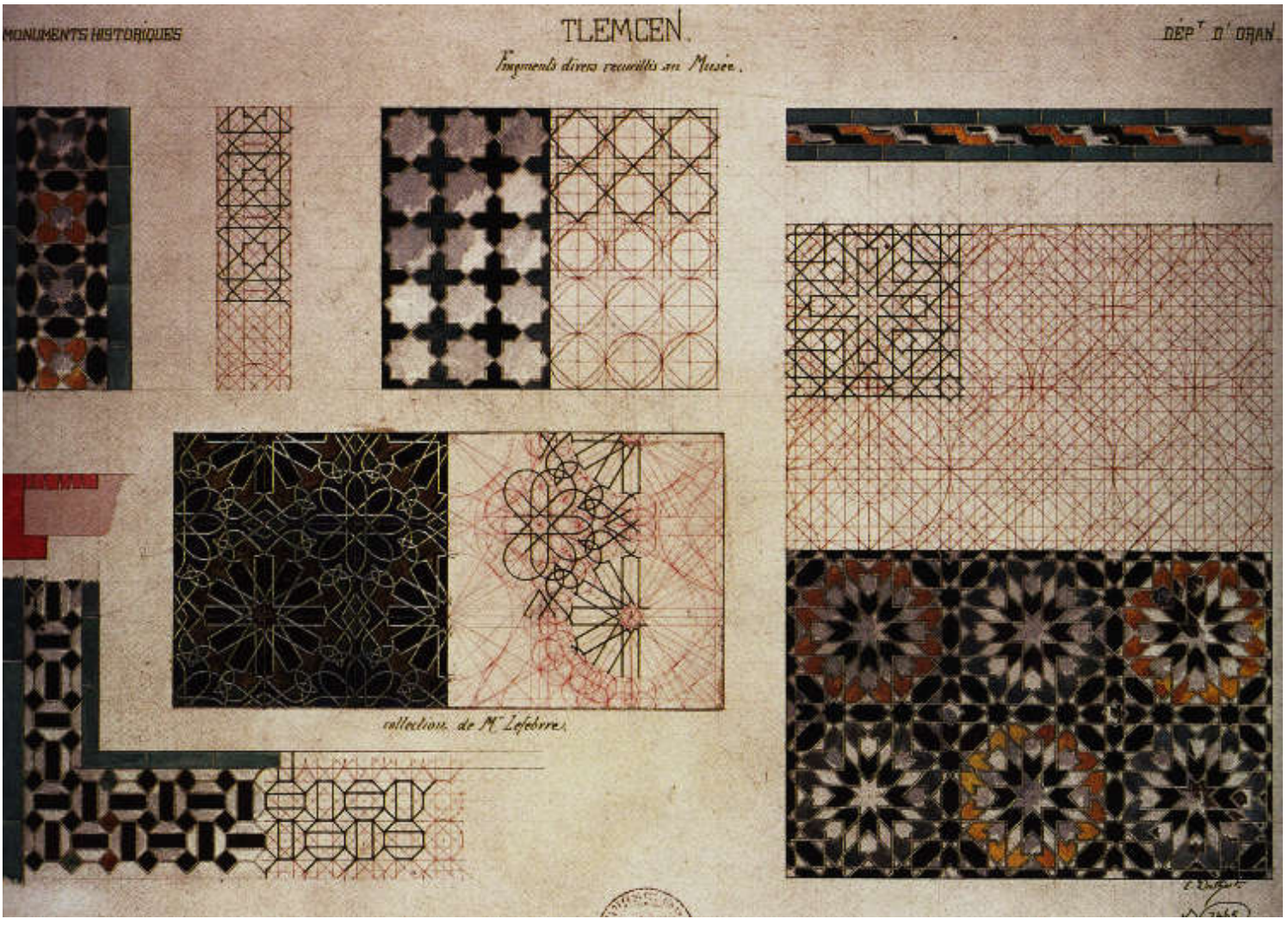

Fig.8. Tracé géométrique du zelidj de la Medersa Tachefinia [15]

Il existe trois systèmes de relation entre les formes qui sont : 
- La triangulation qui est obtenu en dessinant deux cercles avec une courbe constante où chaque cercle croise le centre de l'autre. A travers ça, nous obtenons un angle droit, un triangle équilatéral et un ratio de 1/3 (Fig.9). Si nous continuons à dessiner des cercles en forme de courbe, il ne faudrait que les réitérer cinq fois pour former un hexagone, qui peut être fait comme un carré régulier pour débloquer complètement une surface [16].

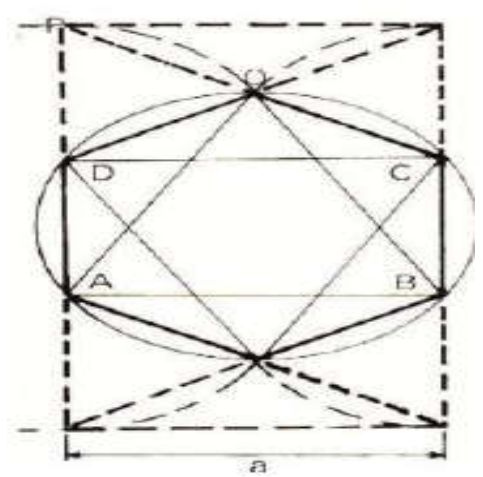

Fig.9. Triangulation [17]

- Les quadratures sont des systèmes proportionnels les plus couramment utilisés et flexibles, car elles permettent à plusieurs groupes d'entiers dans les lignes horizontales et verticales (Fig.10). Les lignes d'organisation diagonale pourraient également être réalisées dans le système de quadrature. Les proportions de quadrature donnent généralement une impression de calme, de bien-être et de satisfaction de la vie. Les proportions de quadrature existent dans les ruines romaines, dans les civilisations du moyen âge jusqu'à l'âge gothique ainsi que dans les civilisations indiennes, bouddhistes et islamiques [18].

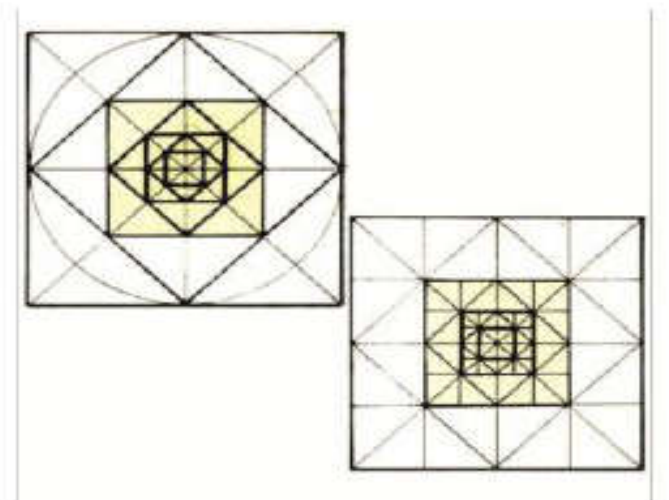

Fig.10. Quadrature [17] 
- Le système pentagonal est basé sur la forme à cinq cotés (Fig.11) qui est la proportion de la moyenne dorée et de l'ambiguïté. Cette forme était le plus grand secret de l'un des pythagoriciens qui est Luca Pacioli, la divine proportion. Il existe des énigmes mathématiques et physiques, des secrets de traditions culturelles entourant le pentagone et le pentagone régulier étoilé [18]

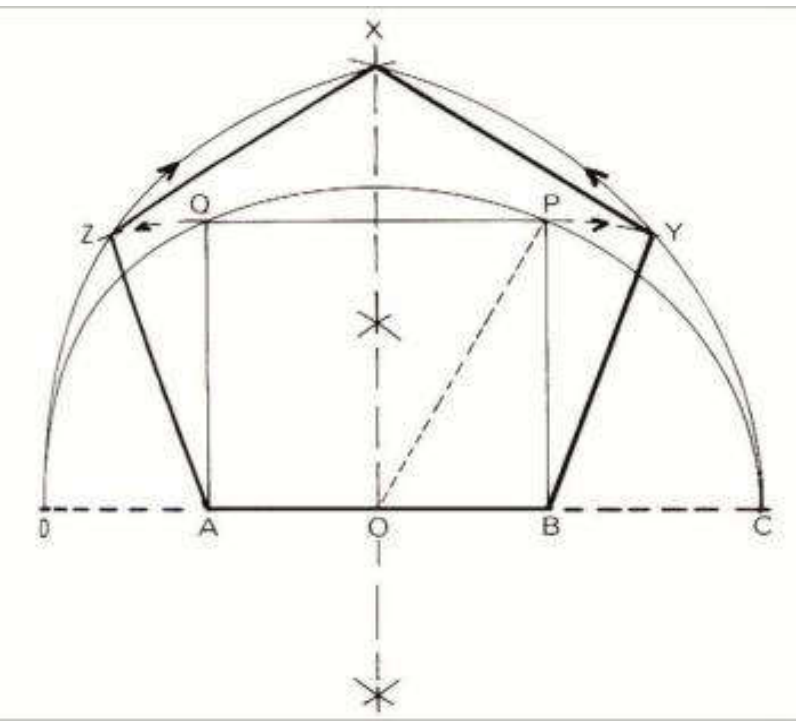

Fig.11. Système pentagonal [17]

En plus, les plans comprenaient des lignes d'organisation (Fig.12). La réalisation des notions de typicité et d'unité se fait à travers les processus de conception. Le plus important parmi ces processus fonctionne sur les lignes principales. Cela comprend la création d'une matrice de lignes avec un espace de trois dimensions visant à vider la forme totale du bâtiment et les relations des éléments constitutifs les uns avec les autres, de sorte que les linéaires identifient l'épaisseur et les emplacements des murs, ou les éléments similaires, taille des trous de construction et l'espace entre les éléments portant le poids. Ainsi, ils établissent le nombre requis, les dimensions et les relations entre elles [19]. Les linéaments sont rarement connectés, et la lecture n'est pas une solution pour le casse-tête puisque nous sommes tous ensemble avec une capacité inconsciente à les distinguer et à les identifier. Les lignées les plus courantes qui sont liées aux constructions principales du bâtiment sont les principaux points de connexion diagonaux et les diamètres de construction, outre les longueurs principales de la construction où l'œil relie les éléments placés le long de ces lignes [20]. 


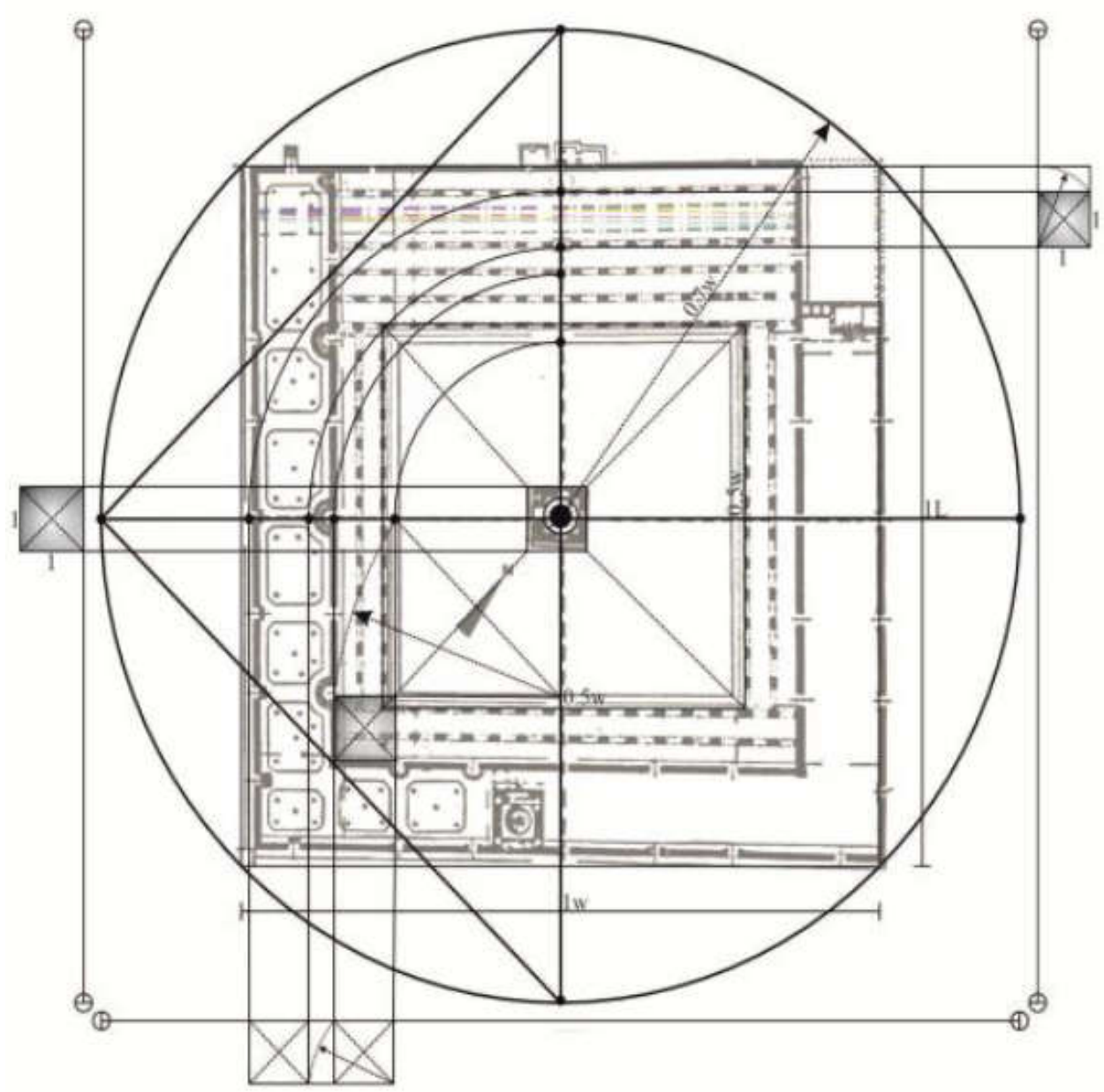

Fig.12. Plan de la mosquée Ibn-Tulun au Caire [16]

Enfin, d'autres principes de composition géométrique sont utilisés comme la symétrie (Fig.13), l'asymétrie, la dissymétrie, l'équilibre, le rythme (Fig.14), la répétition (Fig.15), la hiérarchie, le dégradé la dominance (Fig.16) et la trame [21,22].

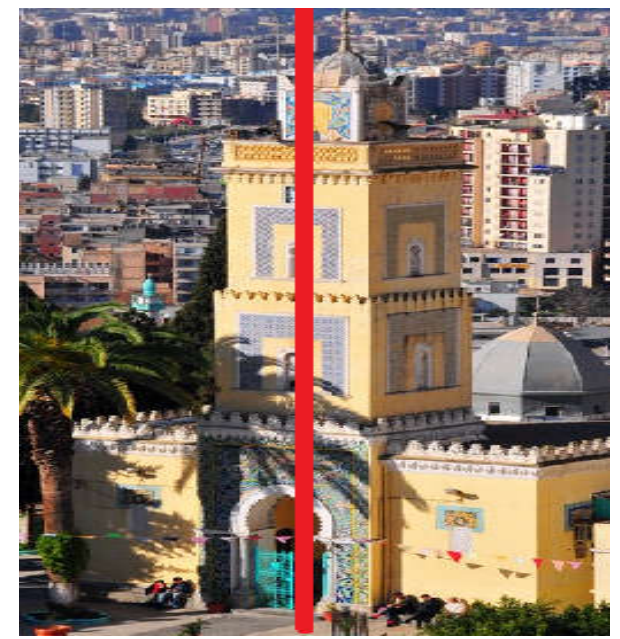

Fig.13. Mosquée Sidi Soufi de Béjaia

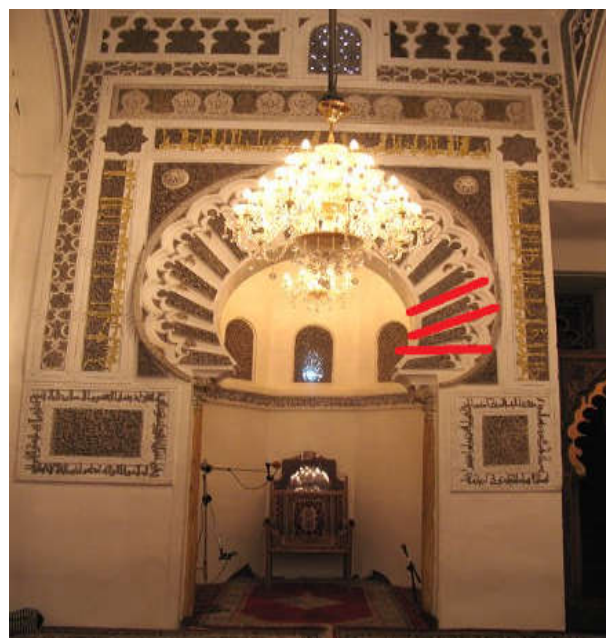

Fig.14. Mihrab de la grande mosquée de Tlemcen 


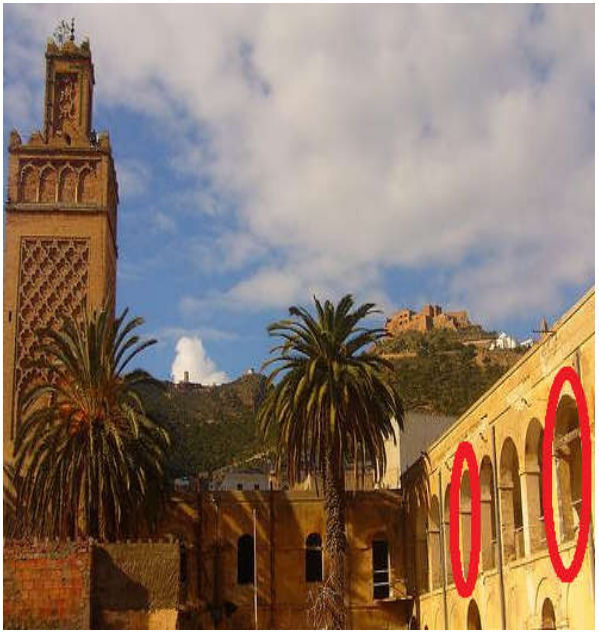

Fig.15. Mosquée Sidi El Haouari d'Oran

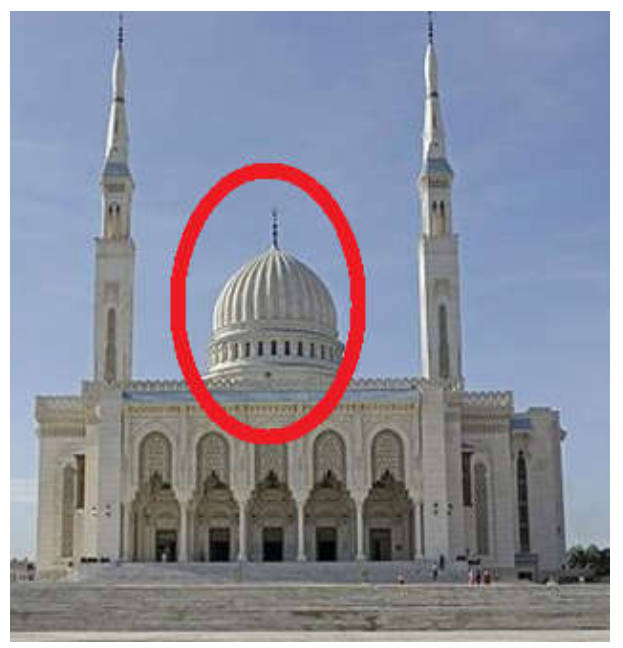

Fig.16. Grande mosquée de Constantine

A l'instar des villes du monde islamique, Tlemcen contient de nombreux édifices présentant ce type de style architectural [23-25] qui témoignent du passage de plusieurs dynasties musulmanes qui sont les Idrissides entre 970 et 1078, les Almoravides entre 1078 et 1147, les Almohades entre 1147 et 1236, les Zianides entre 1236 et 1509, les Mérinides entre 1299 et 1358 et les Ottomans entre 1517 et 1833 [26-28].

\subsection{Problématique}

$\mathrm{Vu}$ par satellite, le plan de la médina de Tlemcen à l'air d'avoir une forme organique (naturelle et quelconque) sans principe géométrique précis. Nous nous sommes interrogés alors sur l'application des proportions géométriques dans les bâtisses de cette ancienne ville. Pour vérifier ça sur terrain, nous avons choisi un monument mineur, qui est la mosquée de Sidi El Bena (date du $14^{\text {ème }}$ siècle à l'époque zianide) et qui a l'air d'être construite d'une façon simple, ce n'est pas comme la grande mosquée, Nedroma, Sidi Bel Hassen, Sidi Brahim, Sidi Boumedienne et Sidi El Haloui ou autre monument majeur. L'ensemble de ses mosquées ont été étudiées par Duthoit, Golvin [29], Marçais [30], Bourouiba [31] et Grandet [32]. Un monument mineur de Tlemcen n'a jamais été étudié auparavant ce qui justifie notre choix.

\subsection{Présentation du cas d'étude}

La mosquée de Sidi El Bena est située au centre-ville de Tlemcen et prend position en plein quartier d'El Kissaria (Fig.17). Cette ancienne bâtisse du $14^{\text {ème }}$ siècle a subi durant la période coloniale (avant 1962) des atteintes à son authenticité où le toit qui était en charpente en bois 
a été transformé en dalle plate en solives métalliques. Sa superficie est de $294 \mathrm{~m}^{2}$ et son gabarit est de RDC. Elle est constituée d'une salle de prière, une Maksourah, des sanitaires, une salle d'ablution, une galerie, un patio, un minaret et un local réservé à l'apprentissage du coran.

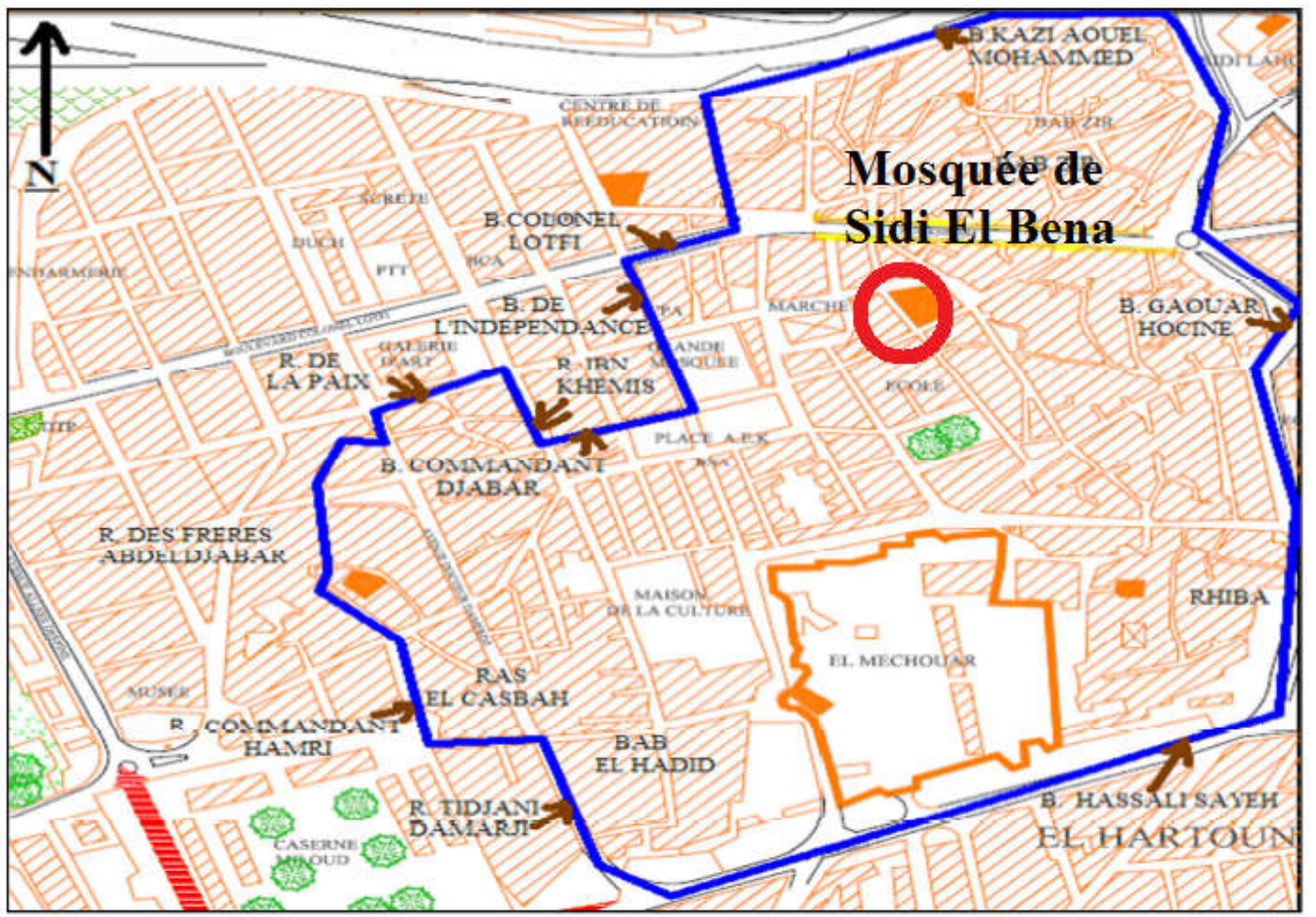

Fig.17. Situation de la mosquée Sidi El Bena

\subsection{Méthodologie}

Afin de répondre à notre question de départ, nous avons tout d'abord pris des relevés des plans et des façades de la mosquée de Sidi El Bena. Ensuite, nous avons comparé entre les mesures des hauteurs, largeurs, longueurs et angles des différents éléments architecturaux pour pouvoir trouver des proportions arithmétiques ou harmoniques tel que le nombre d'or $\Phi$. Enfin, nous avons cherché d'autres principes de composition géométrique cités dans l'introduction.

\section{RESULTAS ET DISCUSSION}

En ce qui concerne le minaret (Fig. 18), nous avons remarqué que la hauteur totale $(\mathrm{H})$ est 
égale à la circonférence $(\mathrm{C})$ ou à quatre fois le coté $(\mathrm{A})$, la hauteur du lanterneau (h) est égale à un côté de la base carrée du fût du minaret (A). Le coté de sa base (a) est égale au 1/4 du côté de la grande base du minaret. La circonférence de sa base (c) est égale aussi à sa hauteur (h). Si nous divisons se coté (a) par deux, nous allons trouver l'unité de base de mesure de l'époque qui est la coudée (c'), qui est égale à $0.4375 \mathrm{~m}$, donc la hauteur du minaret est égale à 32 coudées $(14 / 0.4375)$ et la hauteur du lanterneau à 8 coudées $(3.5 / 0.4375)$. Ces proportions sont justifiées par les formules mathématiques suivantes :

$\mathrm{H}=\mathrm{C}=4 \times \mathrm{A}=4 \times 3.5=14 \mathrm{~m}$

$\mathrm{h}=\mathrm{A}=\mathrm{H} / 4=3.5 \mathrm{~m}$

$\mathrm{a}=\mathrm{A} / 4=3.5 / 4=0.875 \mathrm{~m}$

$\mathrm{c}=\mathrm{h}=4 \times \mathrm{xa}=4 \times 0.875=3.5 \mathrm{~m}$

$c^{\prime}=\mathrm{a} / 2=0.4375 \mathrm{~m}$

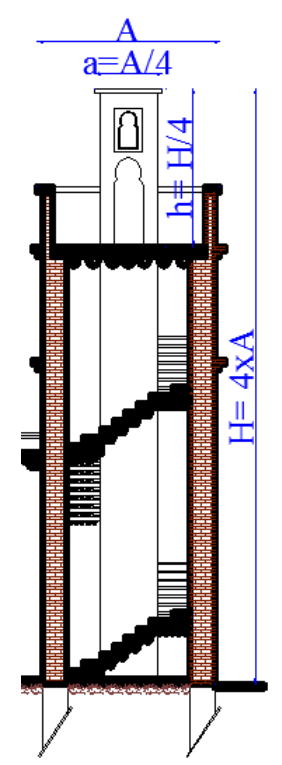

Fig.18. Coupe sur le minaret

Le panneau de la façade du minaret (Fig. 19) est divisé en trois parties, la hauteur de la première (A1) est égale à deux fois le coté de la base du minaret (A). La hauteur de la deuxième (A2) c'est la moitié de la première. La hauteur de la troisième (A3) c'est la moitié de la deuxième, cette dernière distance est égale à deux codées ( $c^{\prime}$ x 2 ). Nous remarquons aussi la même chose pour les arcs décoratifs : le diamètre du petit arc (D) du lanterneau est égale à la moitié d'une codée soit $0.218 \mathrm{~m}$, le diamètre des trois arcs (D1) de la deuxième 
partie du minaret est égale à deux fois celui de l'arc du lanterneau, soit une codée, le diamètre du grand arc de la première partie est égale à son tour à quatre fois le diamètre du petit arc, soit deux coudées. Ceci est justifié par les formules mathématiques suivantes :
$\mathrm{A} 1=\mathrm{A} \times 2=3.5 \times 2=\mathrm{c}^{\prime} \times 16=0.4375 \times 16=7 \mathrm{~m}$
$\mathrm{A} 2=\mathrm{A} / 2=3.5 / 2=\mathrm{c}^{\prime} \mathrm{x} 4=0.4375 \times 4=1.75 \mathrm{~m}$
$\mathrm{A} 3=\mathrm{A} 2 / 2=1.75 / 2=\mathrm{c}^{\prime} \times 2=0.4375 \times 2=0.875 \mathrm{~m}$
$\mathrm{D}=0.218 \mathrm{~m} \approx \mathrm{c}^{\prime} / 2 \approx 0.4375 / 2 \approx 0.2187 \mathrm{~m}$
$\mathrm{D} 1=\mathrm{D} \times 2=0.436 \mathrm{~m} \approx \mathrm{c}^{\prime} \approx 0.4375 \mathrm{~m}$
$\mathrm{D} 2=\mathrm{D} \times 4=0.872 \mathrm{~m} \approx \mathrm{c}^{\prime} \times 2 \approx 0.4375 \times 2 \approx 0.875 \mathrm{~m}$

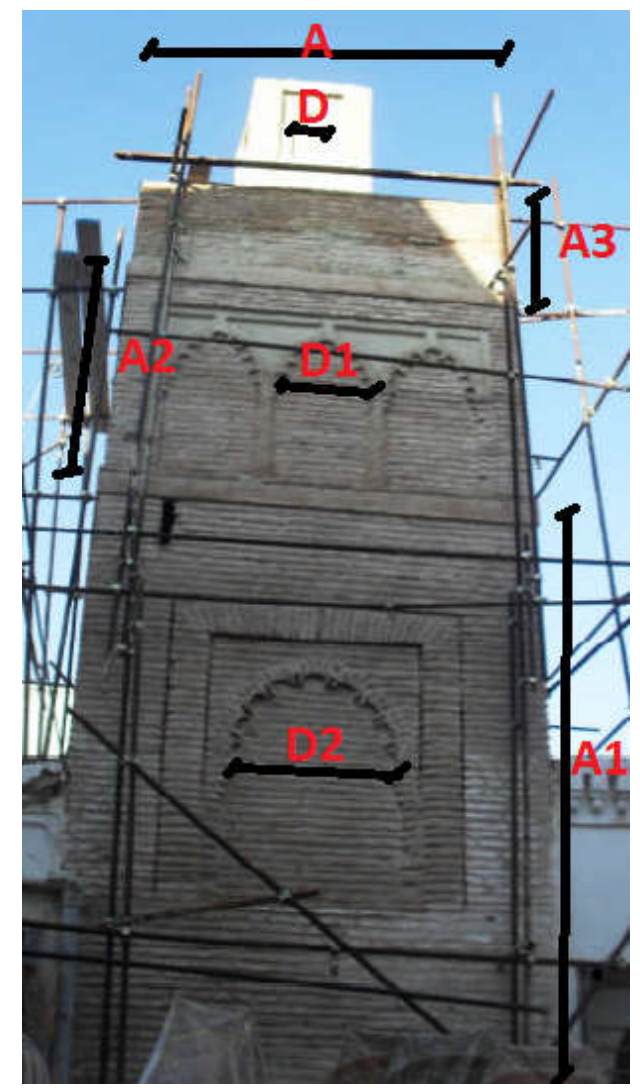

Fig.19. Façade du minaret

Nous trouvons aussi des proportions au niveau du reste de la volumétrie extérieure (Fig. 20 et 21) car la hauteur de la partie réservée à la prière et aux ablutions (H1) est égale à 1.5 fois le coté de la base du minaret, soit 12 coudées (c'). La largeur de cette partie (L1) est égale à la hauteur du minaret $(\mathrm{H})$, soit 32 coudées. Sa longueur est égale à $(\mathrm{L}) 6$ fois le coté du minaret (A), soit 48 coudées. Ces proportions sont justifiées comme suit : 
$\mathrm{H} 1=1.5 \times \mathrm{A}=1.5 \times 3.5=12 \times \mathrm{c}^{\prime}=12 \times 0.4375=5.25 \mathrm{~m}$

$\mathrm{L} 1=\mathrm{H}=4 \times \mathrm{A}=4 \times 3.5=32 \times \mathrm{c}^{\prime}=32 \times 0.4375=14 \mathrm{~m}$

$\mathrm{L}=6 \times \mathrm{A}=6 \times 3.5=\mathrm{H} / 2 \times 3=14 / 2 \times 3=48 \times \mathrm{c}^{\prime}=48 \times 0.4375=21 \mathrm{~m}$

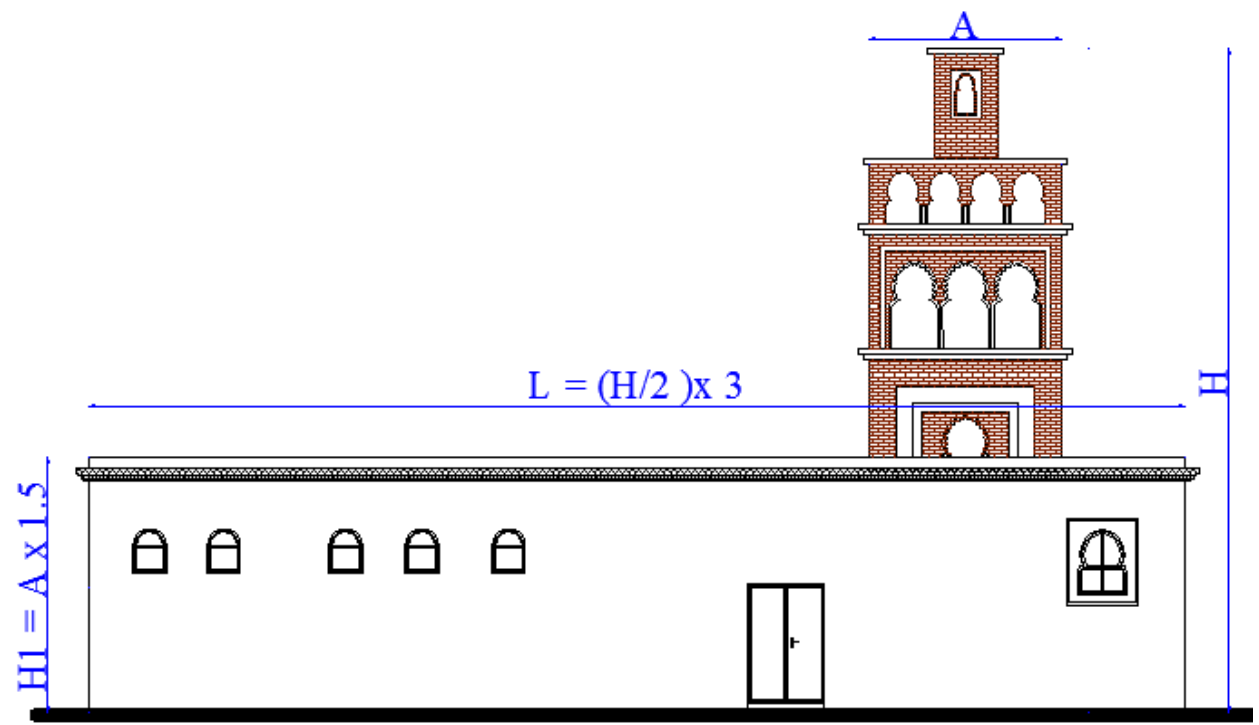

Fig.20. Façade latérale

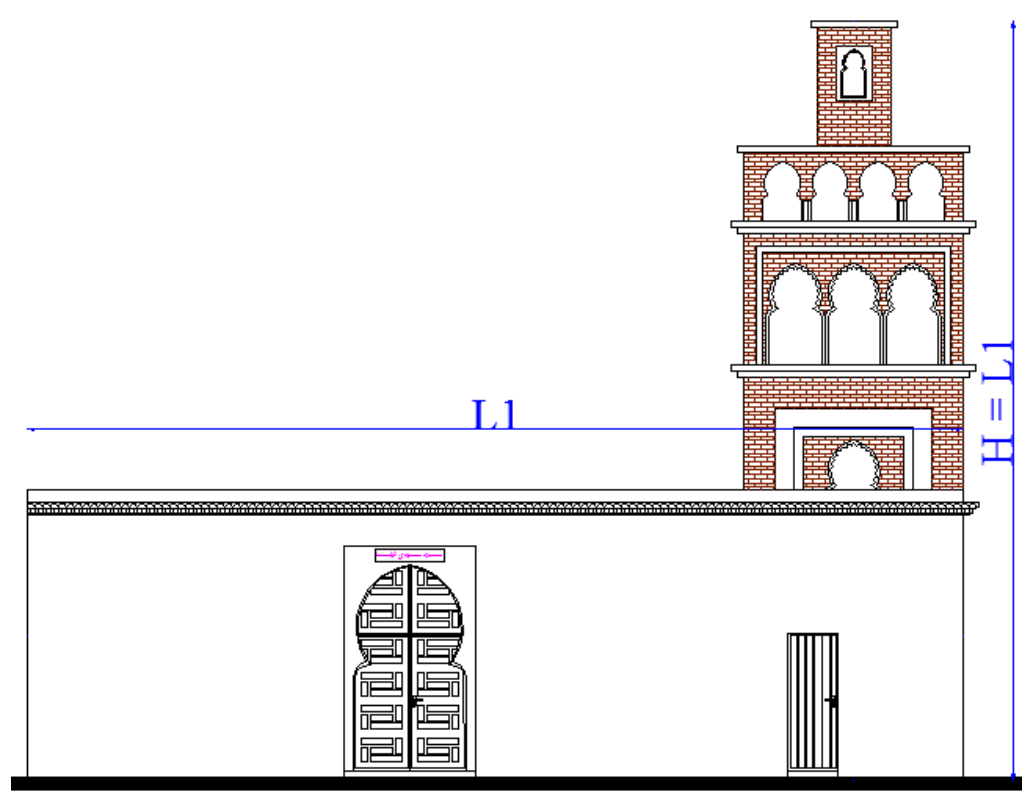

Fig.21. Façade Principale

La division des deux parties (Fig. 22) : prière et ablutions dans le plan de la mosquée est faite suivant une proportion dite d'or, en effet, si nous divisons la largeur de la première partie sur la deuxième, nous allons trouver le fameux nombre d'or $1.618(\Phi)$. Ceci est vérifié suivant la formule suivante : 
$\mathrm{B} / \mathrm{B} /(\mathrm{L}-\mathrm{B})=12.98 /(21-12.98)=\mathrm{B} / \mathrm{C}=12.98 / 8.02=\Phi=1.618$

L1

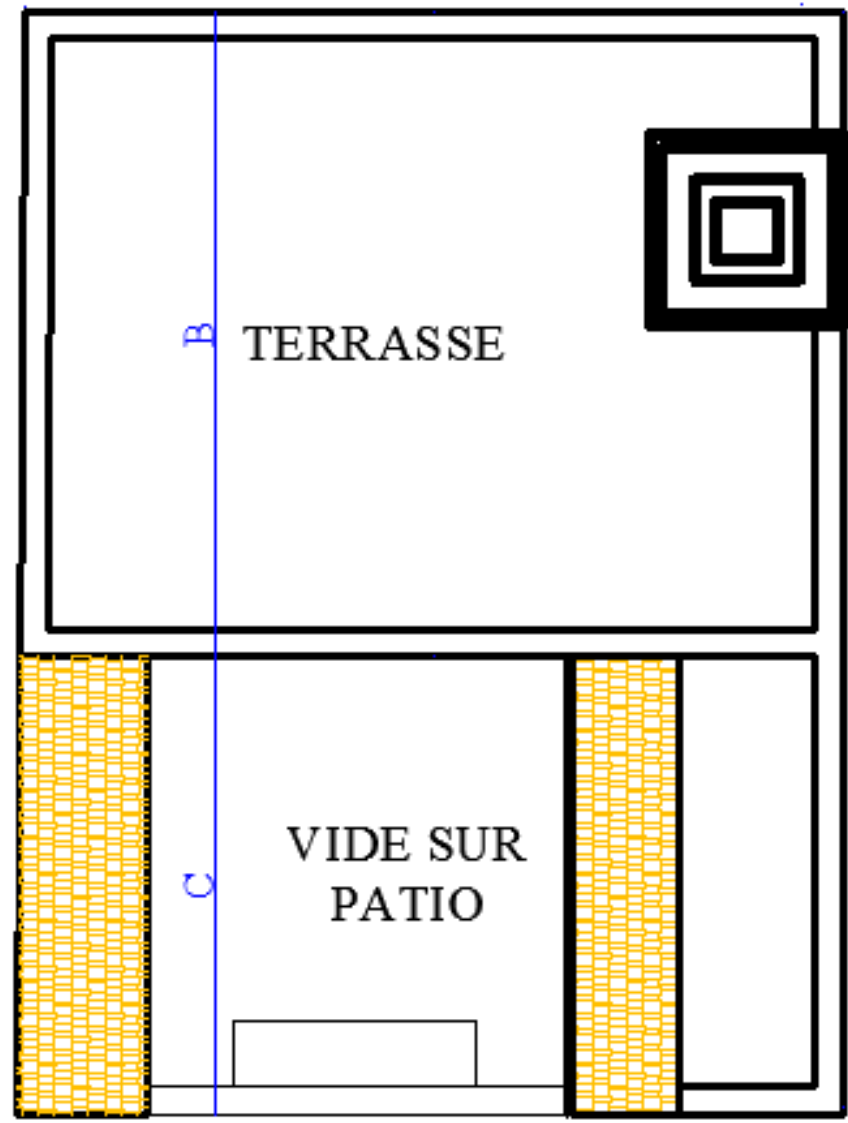

Fig.22. Plan de toiture

Le plan de la mosquée (Fig. 23) s'inscrit dans un rectangle d'or puisque l'angle de la diagonale est égal à $36^{\circ}$ et la formule de vérification nous donne une proportion de $1.618(\Phi)$. Ce rectangle est divisé en deux petits rectangles (partie prière et annexes). Le premier est aussi un rectangle d'or. Le deuxième présente une proportion qui est égale à $\Phi / 2$. Les formules mathématiques suivantes nous confirment cela :

$\Phi=2 \cos (\alpha)=2 \cos \left(36^{\circ}\right)=2 \times 0.809=1.618$

$2 \cos (\beta)=2 \cos \left(66.15^{\circ}\right)=2 \times 0.404=0.808=1.618 / 2=\Phi / 2$

Les ouvertures (fenêtres et portes) ont été mesurées par la coudée, la plus petite présente une coudée ( $\left.c^{\prime}\right)$ et la plus grande présente six coudées $\left(6 c^{\prime}\right)$. La salle de prière est divisée suivant le système de modulation où elle présente trois nefs, la centrale donne sur le mihrab, les trois trames sont égales, leur dimension (E) est de 6 coudées. Sur la largeur la salle présente 4 
trames. Les deux du milieu sont égales aussi à 6 coudées et les deux autres (F) à 4 coudées. Les trames de la salle d'ablution et de la galerie sont égales aussi. Dans le sens de la langueur la dimension est de 3 coudées et dans l'autre sens à 4 coudées. Nous remarquons aussi une symétrie dans l'aménagement des deux parties.

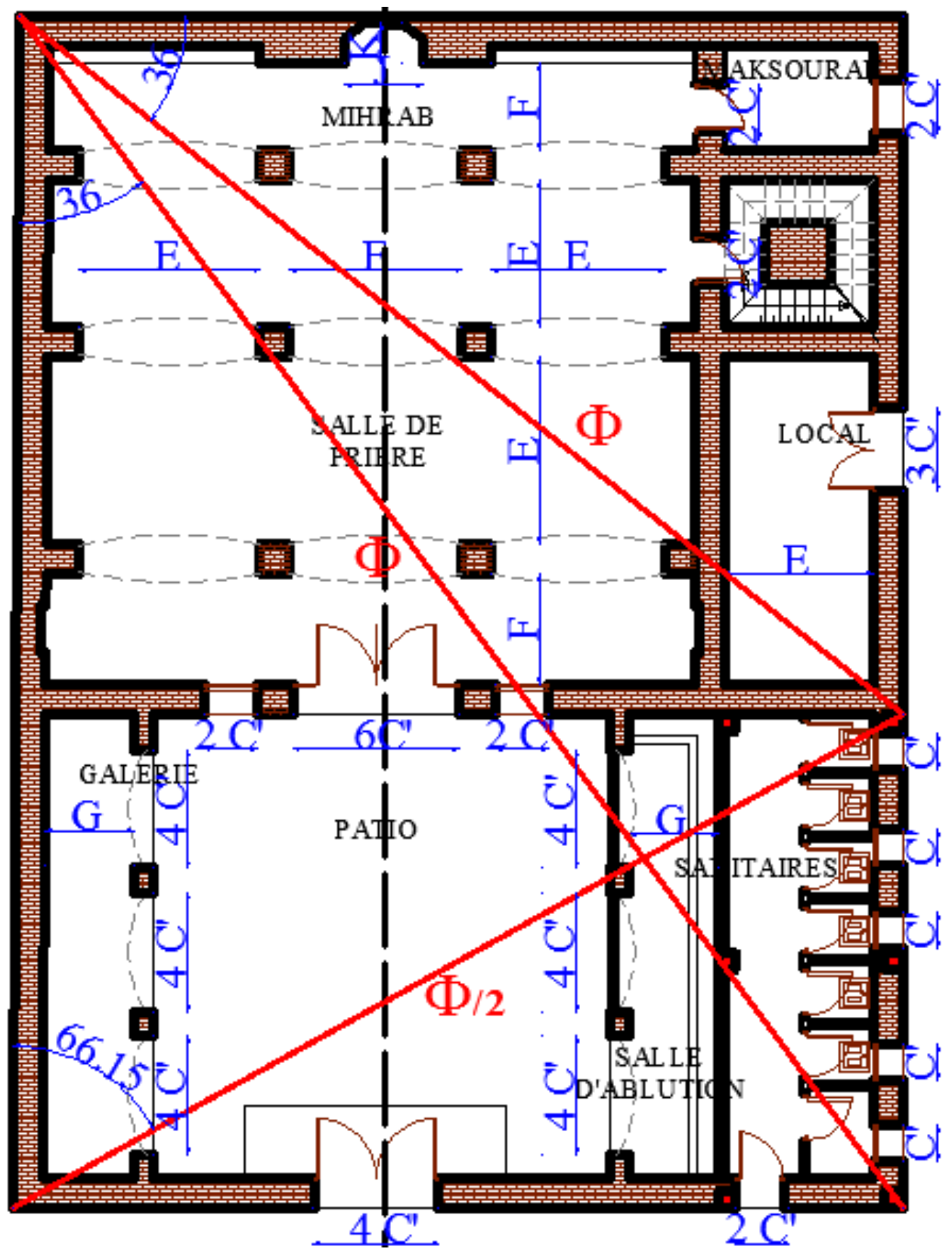

Fig.23. Plan de la mosquée

En ce qui concerne les hauteurs intérieures (Fig. 24), les panneaux des arcs présentent (I) 11 coudées. Chacun est constitué de deux parties la colonne plus son chapiteau et l'arc. Ces deux dernières sont égales (I1), leur dimmention est de 5.5 coudées, soit la moitié de la hauteur 
totale. L'arc est de type outrepassé son rayon (R) mesure 4 coudées. La hauteur du mihrab (I2) est égale au $2 / 3$ de la hauteur du paneau d'arc et son rayon (r) à un coudée, soit $1 / 4$ celui du grand arc. Ces proportions sont vérifiées par les formules suivantes :

$$
\begin{aligned}
& \mathrm{I} 1=4.82 \mathrm{~m} \approx 11 \times 0.4375 \approx 11 \times \mathrm{c}^{\prime} \\
& \mathrm{I} 1=\mathrm{I} / 2=4.82 / 2=2.41 \mathrm{~m} \approx 5.5 \times 0.4375 \approx 5.5 \times \mathrm{c}^{\prime} \\
& \mathrm{I} 2=3.20 \mathrm{~m} \approx 2 / 3 \times \mathrm{I} \\
& \mathrm{R}=1.75 \mathrm{~m}=4 \times 0.4375=4 \times \mathrm{c}^{\prime} \\
& \mathrm{r}=0.43 \mathrm{~m} \approx \mathrm{R} / 4 \approx 1.75 / 4 \approx 0.4375 \approx \mathrm{c}^{\prime}
\end{aligned}
$$

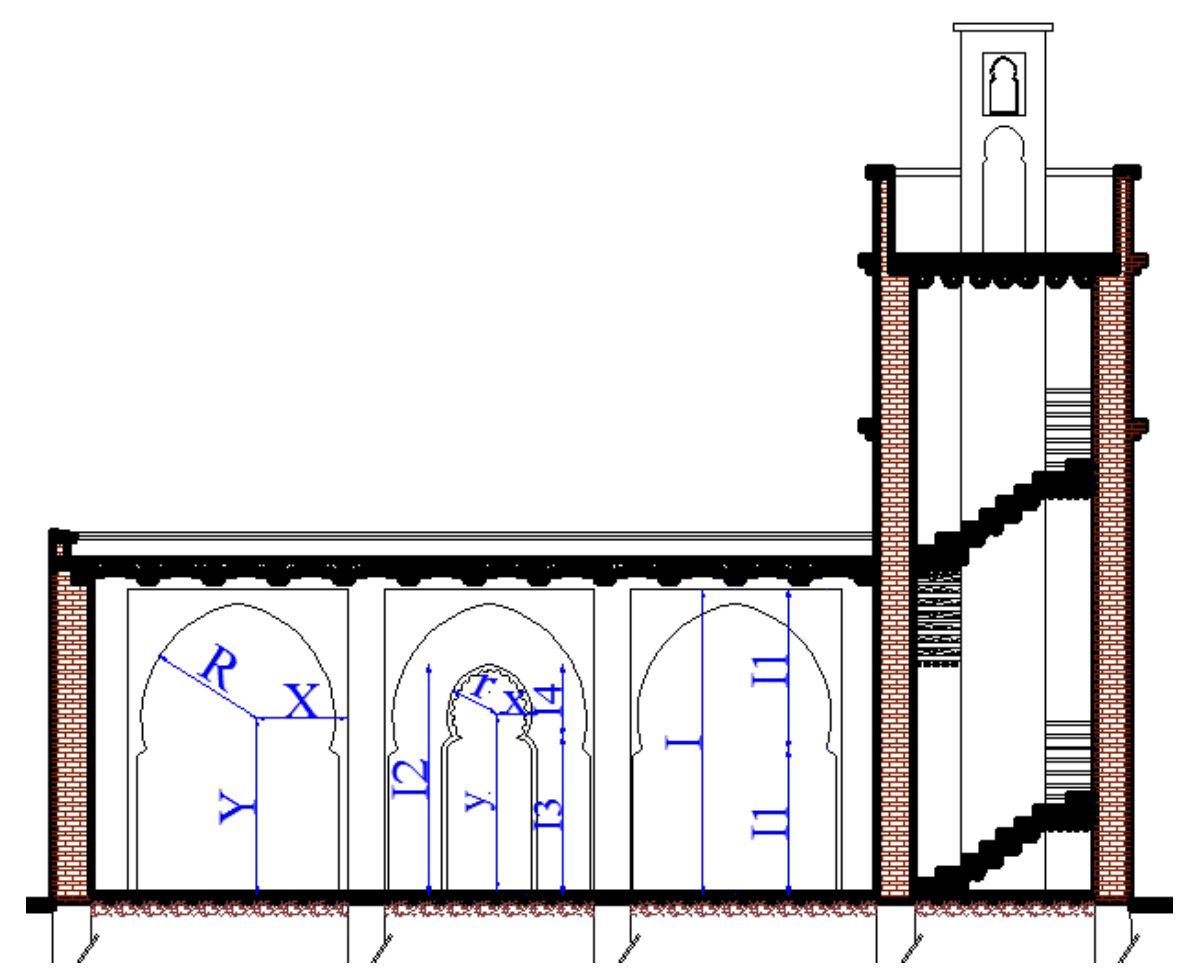

Fig.24. Coupe sur la mosquée

Les autres principes de composition géométriques en plus de la symétrie sont présents telles que la réduction d'échelle des arcs et la répétition des éléments architecturaux comme les arcs, les denticules des corniches et les fenêtres. Le décor n'a pas été traité dans cet article car la mosquée a perdu ses arabesques, muqarnas et zelidj lors d'un incendie durant l'occupation française. 


\section{CONCLUSION}

A travers notre étude des proportions géométriques, nous avons trouvé que l'architecture de la mosquée zianide de Sidi El Bena présente de nombreuses proportions arithmétiques entre les dimensions de ses éléments architecturaux. Les proportions harmoniques sont aussi présentes surtout dans la décomposition de ces espaces, nous avons pu alors trouver le fameux nombre d'or $\Phi$. L'usage du module est présent notamment dans la trame structurelle. Notre calcul nous a permis de trouver la dimension exacte de la coudée de l'époque qui est égale à $0.4375 \mathrm{~m}$, elle est presque égale à celle des romains $0.444 \mathrm{~m}$. D'autres principes de composition géométrique sont utilisés telles que les répétions et la symétrie.

\section{REMERCIEMENTS}

Nous remercions infiniment les Directeurs des Directions de la Culture, des Affaires Religieuses et Waqfs qui nous ont donné l'autorisation de prendre les relevés de la mosquée de Sidi El Bena. Nous remercions aussi l'imam de la mosquée pour ses explications qui nous ont été bénéfiques pour la rédaction de notre article.

\section{REFERENCES}

[1] Dabbour L. M. Geometric proportions: The underlying structure of design process for Islamic geometric patterns. Frontiers of Architectural Research, 2012, 1(4):380-391.

[2] Yahya Abdullahi Y., Bin Embi M.R. Evolution of Islamic geometric patterns, Frontiers of Architectural Research, 2013, 2(2):243-251.

[3] Kiani Z., Amiriparyan P. The Structural and Spatial Analysing of Fractal Geometry in Organizing of Iranian Traditional Architecture. Procedia - Social and Behavioral Sciences, 2016, 216(6): 766-777.

[4] Hejazi M. Geometry in nature and Persian architecture. Building and Environment, 2005, 40(10):1413-1427.

[5] Cromwell P. R. Islamic geometric designs from the Topkap1 Scroll I: unusual arrangements of stars. Journal of Mathematics and the Arts, 2010, 4(2): 73-85.

[6] Cromwell P. R. Hybrid 1-point and 2-point constructions for 
some Islamic geometric designs. Journal of Mathematics and the Arts, 2010, 4(1): 21-28.

[7] Cromwell P. R. Islamic geometric designs from the Topkapı Scroll II: a modular design system. Journal of Mathematics and the Arts, 2010, 4(3): 119-136.

[8] Henry R. A Review of "Islamic geometric design”, by Eric Broug. Journal of Mathematics and the Arts, 2015, 9(1): 48-53.

[9] Lozanovska M. Hagia Sofia (532-537AD): A study of centrality, interiority and transcendence in architecture. The Journal of Architecture, 2010, 15(4): 425-448.

[10] Büyükdiğan I. Muqarnas Domes in Edirne. Architectural Science Review, 2001, 44(2): $161-171$.

[11] Goodwin G. The art and architecture of Islam 1250-1800. Asian Affairs, 1996, 27(2): $177-256$

[12] Lakhi C. M. Islamic Aesthetics: A Re-Assessment 1969, De arte, 4(6): 50-63.

[13] Islam I., Noble A. Mosque Architecture in Bangladesh: The Archetype and Its Changing Morphology. Journal of Cultural Geography, 1998, 17(2): 5-25.

[14] Hyman A., Myer W. Book Reviews Arts of the Islamic world. Central Asian Survey, 1996, 15(2): 299-317.

[15] Duthoit E. Tracé géométrique du Zelidj de la Medersa Tachefinia. Médiathèque de l'Architecture et du Patrimoine, Paris, 1873.

[16] Kadhim F. K., Julaihi W. The proportional relations systems of Islamic architecture. International Journal of Scientific and Research Publications, 2013, 3(1):1-12.

[17] El-Said, I., Parman, A. Geometric Concepts in Islamic Art. Scorpion Publishing Limited World of Islam Festival Trust, Guildford, England, 1976.

[18] AliAbadi M. Eternal geometry (or geometry of the sky) in Islamic architecture, International Journal of Engineering Science, 2007 18(5): 335-357.

[19] Crowe N. Nature \& the idea of a man - made World: An investigation into the evolutionary roots of form and order in the built environment. The M.I.T. Press, Cambridge, Massachusetts, London, 2003.

[20] Ganizadeh Hesar N., Najaf Golipour Kalantari N., Ahmadi M. Study of Sacred Geometry in Islamic Architecture. Cumhuriyet Science Journal (CSJ), 2015, 36(3):3800-3812. 
[21] MahdiNejad J, Sadeghi HabibAbad A. Expressions of Islamic ideas and foundations in the noble iranian-islamic architecture, (case study: khan school in shiraz). J. Fundam. Appl. Sci., 2016, 8(3):1115-1131.

[22] Benarbia I. L'évaluation de la valeur esthétique des monuments historiques, mémoire de magister. Université de Tlemcen, Algérie, 2012, 74p.

[23] Hamma W., Djedid A., Ouissi M. N. Intervention on historical city Tlemcen during 1960-2009: Impacts and applicability. Urbanism Architecture Constructions, 2016, 7(2):109-124.

[24] Hamma W., Djedid A., Ouissi M. N. Délimitation du patrimoine urbain de la ville historique de Tlemcen en Algérie. Cinq Continents, 2016, 6(13):42-60

[25] Hamma W. Classification and Recognition of the Heritage Values of the Monuments of Tlemcen. Urbanism Architecture Constructions, 2017, 8(2):129-142.

[26] Selka C., Oussadit I., Ouissi M. N., Carrio J. M., Hamma W. Interventions and uses of the defensive heritage in Tlemcen: Case of Bab El Quarmadin. Urbanism Architecture Constructions, 2017, 8(3):221-338.

[27] Hamma W. The Berber buildings destroyed during the French Colonial period in Tlemcen. Urbanism Architecture Constructions, 2017, 8(3):275-282.

[28] Hamma W. Method of rehabilitation of old urban fabrics of Tlemcen. Urbanism Architecture Constructions, 2017, 8(4):315-330.

[29] Golvin L. Essai sur l'architecture musulmane, Klincksieck, Paris, 1970

[30] Marçais G.., Marçais W. Les monuments arabes de Tlemcen. Édition Fontemoing A, Paris, 1903.

[31] Bourouiba R. L'Art religieux musulman en Algérie. SNED, Alger, 1973.

[32] Grandet D. Architecture et urbanisme islamiques. Office des publications universitaires, Alger ,1986.

\section{How to cite this article:}

Hamma W. Geometric proportions in Islamic architecture: case of the Sidi El Bena mosque in Tlemcen. J. Fundam. Appl. Sci., 2017, 9(3), 1435-1453. 